\title{
Weaving Enclosure. Material computation and novel forms of crafting
}

\author{
- Roberto Naboni \\ ACTLAB - Politecnico di Milano, Department \\ $\mathrm{ABC}$, Italy \\ roberto.naboni@polimi.it \\ - Luca Breseghello \\ ACTLAB - Politecnico di Milano, Italy \\ luca.breseghello@mail.polimi.it
}

\begin{abstract}
This paper presents a computationally based methodology arising from the application of weaving techniques for the design and construction of an experimental architectural system, Weaving Enclosure. The research explores the close correspondence between material properties and assembly systems found in the traditional craft of weaving, studied through analytical and laboratory tests, and then implemented through computational design and digital fabrication. The workflow for the generation of specific geometries related to the elastic nature of the material results in the design and fabrication of a self- standing interior partition with digitally conceived patterns, tuned parametrically to provide structural and screening performance.
\end{abstract}

Keywords: Computational Design, Digital Crafting, Digital Fabrication, Material Computation, Weaving

\section{Introduction}

Contemporary research in architecture, favoured by the use of advanced computational tool-sets, has focused on the integration of material properties within the digital design environment in order to inform physically consistent architectural design. This approach enables the designer to embed complex material characteristics and behaviours as an active agent in the making of architecture (Menges 2012) and allows the exploration of non-standard building materials and techniques. The industrial needs for standardized production processes have selected just a limited number of techniques considered appropriate to satisfy the criterias for mass production. With the implementation of custom-based digital workflows, antique craft-based techniques can be revisited and reinterpreted under the perspective of "digital crafting", attempting at defining a novel relationship between material behaviors and human activities, mediated through the use of computational tools, working on innovative interpretations of old processes, performance and language towards their implementation in architecture (Oxman 2007; Thomsen and Tamke 2014).

Weaving in architecture. Origins and contemporary applications

The technique of weaving has been widely acknowledged as one of the most antique surviving crafts - the oldest known woven baskets, indeed, have been carbon dated to between
10,000 and 12,000 years old (Wikipedia, Basket Weaving). It is a cross-cultural phenomenon found in almost everywhere throughout the globe. The patterns and specific techniques normally vary from one place to another but they share a common knowledge based on the inherent properties of the materials used. A woven artifact can be considered the result of mediation between material and humans, between raw products of a territory and the cultural knowledge of craftsmanship. A sort of manual material computation is indeed implemented while working with the raw material. Through a direct engagement with matter, weavers perform an immediate problem-solving procedure, as every knot they make constrains the next weaving step (Muslimin 2010).

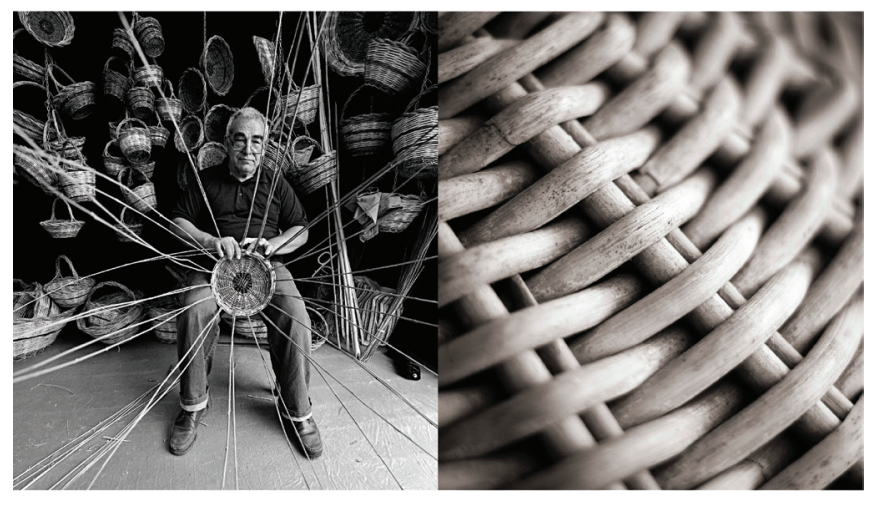

Figure 1: Traditional basketry weaving system. 
The antique use of weaving in architecture was first described by Gottfried Semper in 1851 in the book - The Four Elements of Architecture, where the construction of the primitive hut was categorized into four fundamental elements - Hearth, Roof, Mound and Fence (Semper 1989). The Fence refers to the walls of the ancient houses, which Semper described as woven "mats" or hanging cloths rather than solid stone structures. These walls were made out of wooden pieces, branches and plants of varying sizes intertwined in order to form a supportive structure, in some cases covered with mud or leaves for better thermal insulation. This strategy has been used for hundreds of years up to the last century as the latest example of woven walls might be considered the lath and plaster system nowadays replaced by the gypsum board construction (Griffen 2001). According to Frei Otto the facilitated harvesting and the possibility to be manipulated by hand, made living plants like bamboo or branches of broad-leaved trees a proper weaving material used for the construction of the first human dwellings (Otto 1969). Several attempts to repropose weaving in architecture have been developed within the last few years. We can essentially distinguish two approaches. The first one uses traditional weaving patterns to characterize the aesthetic appearance of architectural systems, such as the structural system (Centre Pompidou in Metz by Shigeru Ban) or the building envelope (Aragon Pavilion in Zaragoza by Olano y Mendo Arqitectos). The second approach applies traditional woven materials over the building skin, as in the Spanish Pavilion in EXPO 2010 by EMBT, or in the facade London Guy's Hospital by Thomas Heatherwicks. Most of these works focus on implementing the aesthetic image of the weaving technique while its intrinsic structural and constructive potential is often neglected.

Potential of weaving and possible applications in contemporary design

One of the main reasons why the weaving technique is not utilized for the construction of walls nowadays is the high level of permeability that it exhibits (Muslimin 2010). In the research outlined in this paper this feature has been reconsidered and implemented in the design of a proto-tectonic semi-permeable partitioning system. Furthermore, weaving shows considerable potential for the design and construction of complex doubly curved surfaces, due to the specific bending characteristics of the material. Weaving structures accommodate natural variations in curvature and actually gain morphological rigidity when implemented on curved geometries. Another fundamental characteristic of this technique is its capacity to establish incredibly resistant and complex construction systems through the use of cheap raw material, from roots to grass, from cane to willow. Starting from these premises, the research focused on the creation of an architectural weaving system in which form, structure and material are coherently developed and integrated as in biological formations (Hensel et al 2010; Oxman 2010), taking advantage of the intrinsic mechanical properties of the technique. This research attempts at answering three main questions. First, how can computational tools inform traditional crafting techniques towards the formation of novel architectural systems? Second, how can we accurately compute the complex behaviour of weaving patterns and inform it with performative criteria? Third, how can we mediate between the high precision of digital fabrication tools and processes, and the imperfections inherent to natural materials?

\section{Methodological Procedures}

\section{The material system of weaving}

Technically, weaving is defined as a process of construction by interlacing or interweaving strips or strands of pliable material. Fundamental elements in defining this material system, in order to provide clear specifications within its use in architecture, include several micro and macro aspects, such as the primary constituent material and the study of its properties, the typology of weaving patterns and the overall characteristics of the woven ensemble to be used performatively (Fig.2).

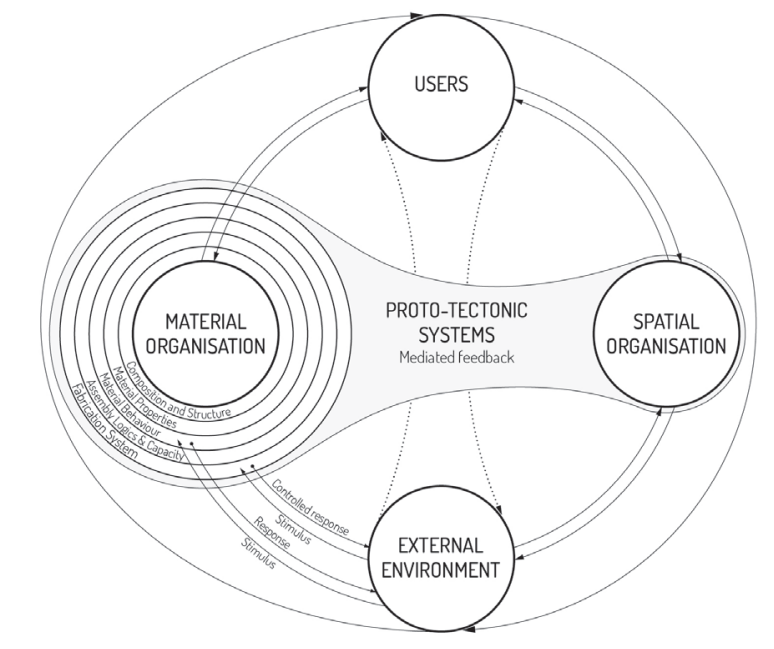

Figure 2: The diagram illustrates how the different features of a material system definition are central in the development of prototectonic systems, which are finally developed in consideration of the wider spatial organization, and conceived as mediation between an external cultural and natural environment and the experience of users (adapted from Hensel 2013).

Initial analysis has been made to define the most appropriate materials for weaving at the scale of architecture. Focusing on natural materials, different species of Willow (Salix) and Rattan, (colloquial name given to a large group of climbing palms) have been analyzed in order to determine the most appropriate material towards the realization of a full scale architectural system. Several families of willow have 
been studied according to their geometrical characteristics and elastic behavior, together with various mechanical tests and FEM analysis (Fig.3). Generally, willow sticks exhibited several limitations as insufficient length and non-constant cross-section, which tends to diminish proportionally towards the top end, made them inappropriate for larger scale applications. Moreover, the section typically has an inner core and irregularities that do not allow an accurate computer simulation of its bending behavior.
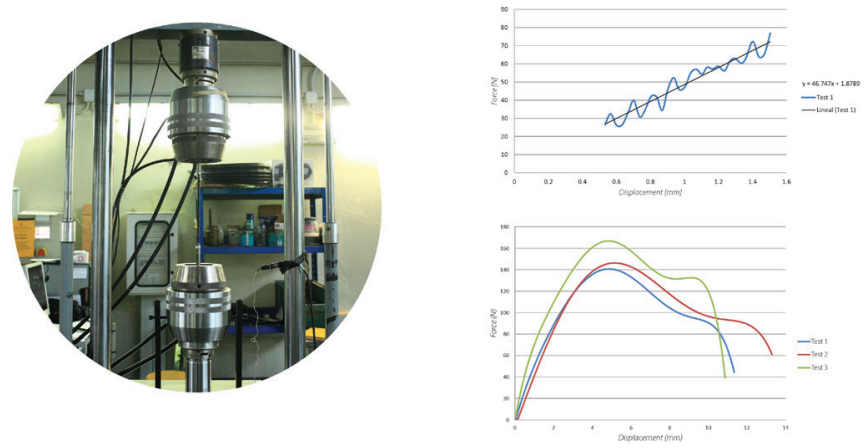

Figure 3: Tensile test on willow. The results evidence strong irregularities between the different tests.

Commercial rattan is generally stronger than traditional European wicker materials (Saunders 1990). It presents an even distribution of vascular bundles over the stem, diversified cross-section consisting of fibers with equal length and equal cap size, conducting cells and ground parenchyma of small cells with polylamellate structure (Dransfield et al. 2000) (Fig.4). These anatomical features and the constant sectional composition and diameter allow for a computational prediction of its behavior. Moreover, its mechanical properties have proved to be relevant for concrete reinforcements (Obilade et al. 2014) as well as for bone replacement, due to its porous structure permitting blood vessels and other accessory tissues to penetrate it, allowing seamless integration into the host bone (Eichenseer et al. 2009). Rattan's considerable length (up to 100 meters) and vascular morphology contribute to define a lightweight material with high tensile properties and elastic bending capabilities. These characteristics of rattan made it a suitable material for the research development.
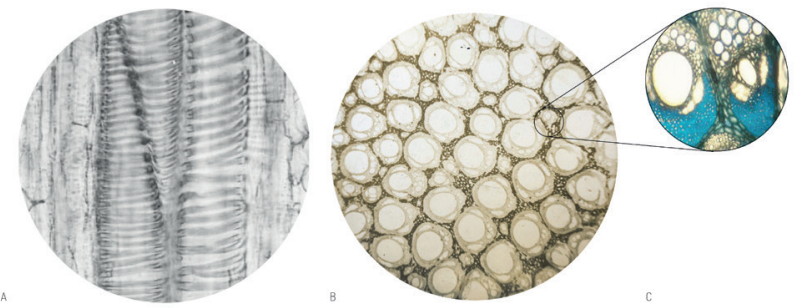

Figure 4: Rattan cellular structure. A. Longitudinal section of protoxylem elements (part of the vascular bundles), (Tomlinson, 2001); B. Transverse section of vascular architecture; C. Vascular bundles with prominent metaxylem vessel (photo J.B. Fisher).

\section{Logics of weaving patterns}

Weaving patterns owe their mechanical performance to the frictional and bending resistance generated by the interlacing sticks. Thus, the interlacing configuration of simple elements produces a highly redundant ensemble with emergent structural properties, which are a direct consequence of the pattern characteristics. A strict correlation between stick typology and weaving pattern does exist, as the choice of the material section naturally reduces the range of pattern variation. For the purpose of the experiment a circular "rod" stick section had been favoured over a plated one. The mechanical properties of each material allow specific operations of twisting or bending. Five typical pattern typologies have been analyzed: Coiling, Twining, Plaiting, Linking \& Looping, Strike \& Strand. Their physical and digital testing, in terms of algorithmic description, have determined the choice of the Strike \& Strand typology which exhibited good mechanical resistance (Fig.6). Furthermore, it is relatively easier to be manufactured and it provides a less constrained bending curvature, offering the opportunity to $o$ be tuned according to extrinsic parameters.

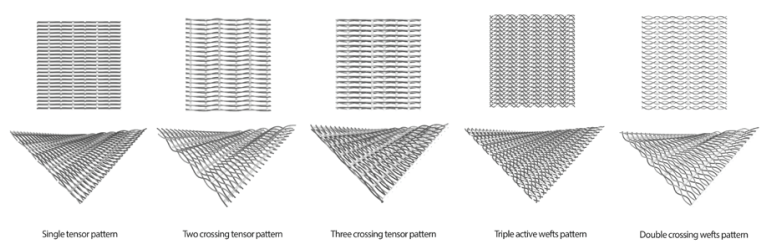

Figure 5: Digital modelling of traditional weaving patterns.
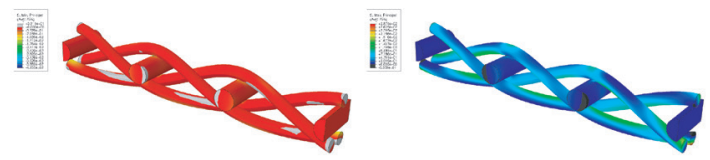

Minimum principal stresses

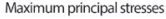

Figure 6: FEM analysis of a typical Strike \& Strand pattern and its Minimum and Maximum principal stresses.

\section{Computational design of weaving patterns \\ Embedding physical properties}

After the selection of the pattern typology, the experiment focused on testing the actual bending behavior of a typical rattan stick and modelling its variable geometrical configurations within the digital environment. This required an understanding of the intrinsic material behavior and finding a convenient way to employ it into the digital model. An accurate simulation of the bending behaviour was obtained through the use of Kangaroo for Grasshopper/ 
Rhinoceros, a well known add-on which embeds physical behaviour directly in the 3D modelling environment. Kangaroo is essentially a Particle-Spring System used for form-finding. Rattan sticks bend elastically within specific limits, which vary in proportion with the level of hydration. This behavior was instrumentally measured determining the minimum elastic bending radius. Wet rattan sticks showed considerably higher bending elasticity, which allowed describing material characteristics in two phases, in dry and wet configurations (Fig. 7).
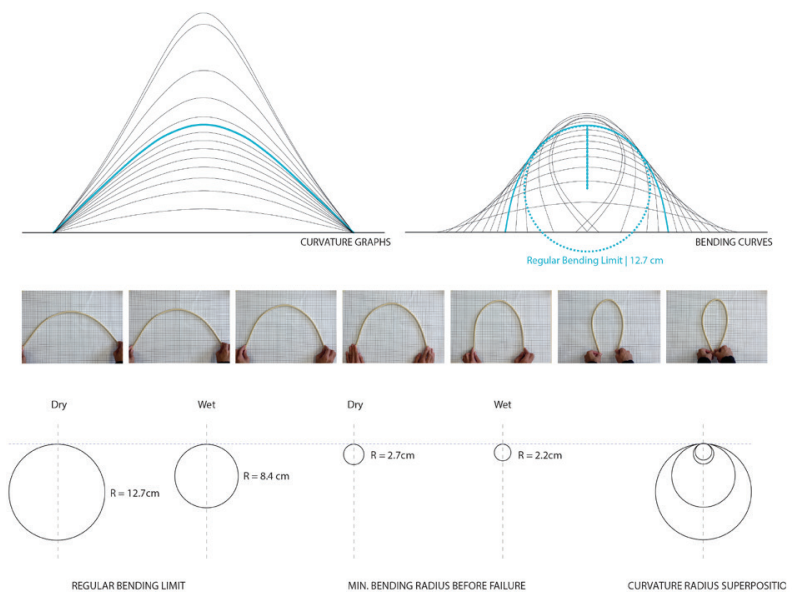

Figure 7: Bending properties of rattan. A. Geometry of bending; B. Radii of rattan bending in dry and wet condition.

Computing large sets of sticks would require multiple running simulations in Kangaroo, and consequently reduce the ability to test and tune different weaving patterns. For this reason a faster modelling method has been developed to evaluate more pattern configurations, making use of B-Spline curves. Splines are originally conceived as curves that closely approximate the shape taken by bending material objects, which minimizes the elastic energy. They are defined by a set of control points, tangents to the curve at these points, and local weighting factors (Fig. 8). These parameters have been precisely set according with the results of the Particle Spring simulation to be finally validated (Fig. 9).

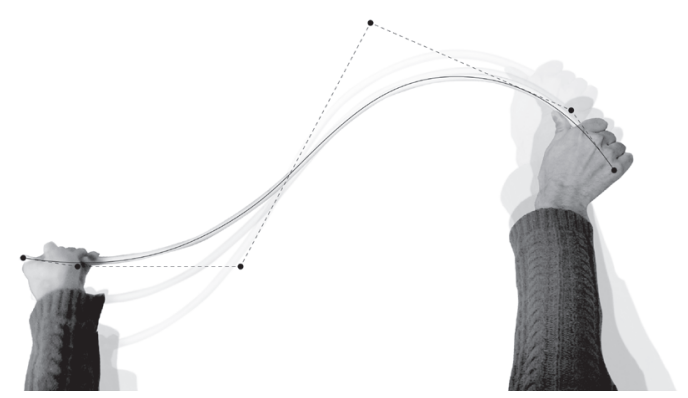

Figure 8: Curvature correspondence between a B-Spline curve and a bent rattan stick through the tuning of its control points.

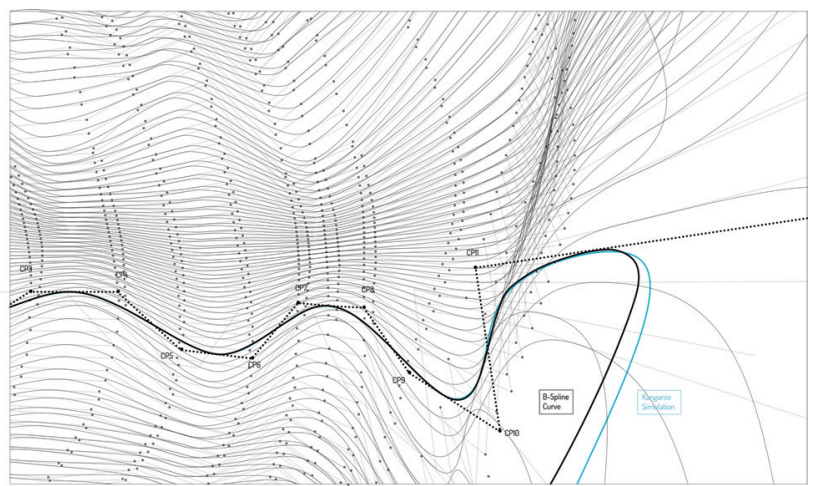

Figure 9: Comparison of bending behavior description between a B-Spline curve,defined by a series of control points, and a curve generated by the Particle-Spring System of Kangaroo.

\section{Design process}

The design experiment was based on a two-directional weaving structure, which is composed by two sets of elements interlaced in a variety of configurations (Ranjan 1985). In this specific case, the vertical warp elements are considered "passive" foundations which define the overall morphological characteristics, while the weft intersecting elements are considered active as they are using bending and frictional strength to confer resistance to the structure. The design process is developed parametrically within Grasshopper. It interprets this structural scheme and embeds the material features following several steps. Starting from an overall sinuous surface (Fig.10a), which is statically stable, a set of poplar plywood warp elements are defined by the discretization of the initial surface and serve as vertical support for the structure (Fig.10b). After temporary horizontal scaffolding are designed (Fig.10c), a rattan weft system, tuned according to differential screening ratios, is generated with the specific rattan curvature characteristics and limits (Fig.10d).

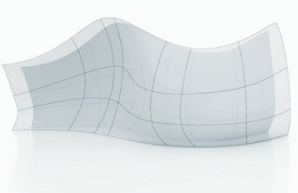
(10a) Morpho-active surface.

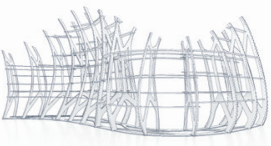

(10c) Construction Skeleton.

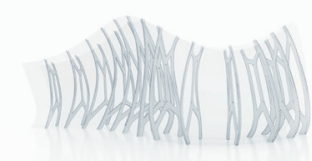

(10b) Plywood Warp Elements- Controlling overall geometry and pattern

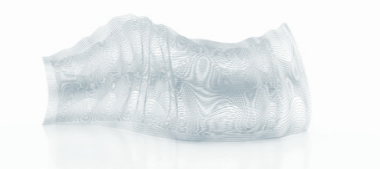

(10d) Rattan Strand-based- Weft System With differential screen ratio.
Figure 10: The complete Weaving Enclosure constructive process. 


\section{Parametric warp system}

A fundamental role in the design of the weaving system is played by the definition of a parametric family of vertical warp elements. Traditional warp elements in weaving are constituted by a single stake element. In this case, shifting to the architectural scale, the single elements have been transformed into a more complex rib formed by a couple of coplanar external stake elements, linked together with an inner support. A shared topological configuration is described by ten control points, which represent a set of parametrically defined links generated in order to optimize the structural rigidity of the single rib, as well as to minimize the material used and to consider the fabrication constraints. Moreover, to further reduce weight, each segment of the rib was parametrically dimensioned according to its length, span and height.

Similar to traditional basketry, the disposition of the vertical warps drives the overall shape of the prototype. Hence controlling the position of the ribs is fundamental to determine overall spatial configurations of the system, adaptable to its final location. The associative definition of the warp system allowed the possibility to vary the form of each single warp element, controlling differential height, width and curvature (Fig.11). The number of ribs, shape and orientation in space are then set within the domain of possibilities previously determined by fabrication and material constraints
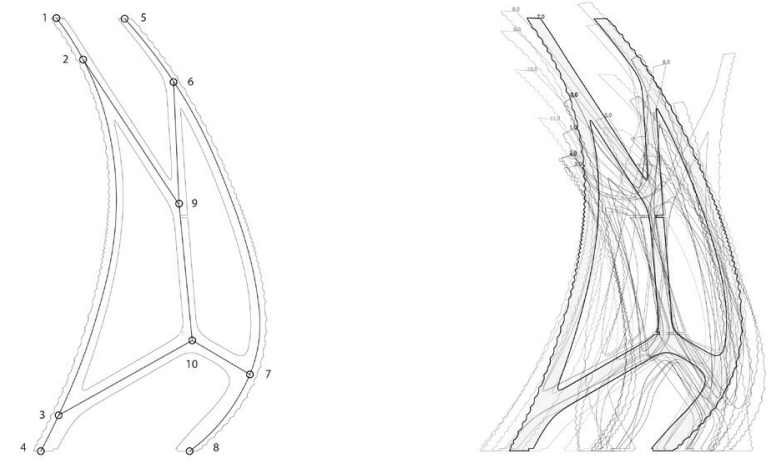

Figure 11: The vertical stakes are conceived as a family of elements which share a common topology defined by ten control points. The associative model allows for variations in order to reflect the needed integration of structural and material behaviors over the shape.

\section{Hybrid fabrication}

\section{Machinic programming}

The prototype has been realized in a hybrid fabrication set, where the plywood warp elements are produced via digital fabrication and the rattan strands are manually assembled. The fabrication was processed through an industrial 3-axis CNC milling machine, which was preventively tested (Fig.12). Several fabrication constraints have been discovered, such as dimensions of the working areas and rubber suction cups to hold the plywood sheets to be placed to ensure milling precision and operational stability of the processed component. These fabrication constraints have been embedded in the original design of the warp elements. To process the cutting paths, typical vectorial files proved insufficient to provide all the needed machining operations. In this case an optimized .cnc format G-CODE was generated from Grasshopper to directly provide the machines with preprocessed working trajectories. This proved to be, during the process, a necessary medium to produce unconventional wooden elements in the case where too many production variables would overcome the ability of an expert operator to handle the wooden production correctly.

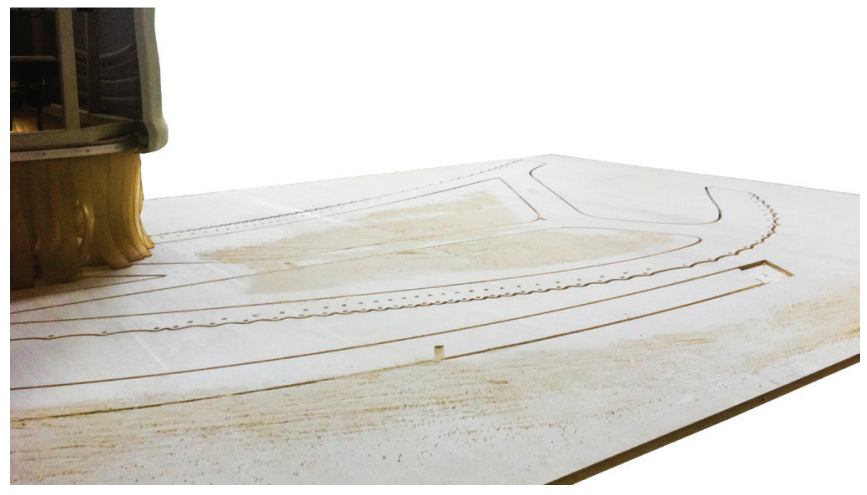

Figure 12: CNC milling of a poplar plywood vertical warp and an horizontal support.

\section{Results}

\section{Material definition}

Different results emerge from the proposed methodology. Investigating the material system highlights that weaving patterns base their mechanical properties on the frictional and bending resistance generated by the interlacing sticks. The physical and digital testing, in terms of algorithmic description, determines the choice of the Strike \& Strand pattern typology which exhibits good and constant mechanical resistance.

\section{Proto-tectonic weaving system}

The previously described weaving material system as well as the computational embedding of its bending behaviour provided a matrix of information which was incorporated within the associative design environment of Grasshopper in order to describe a manifold of possible weaving configurations. Working with this design methodology, the research focused on the design of a proto-tectonic weaving system, as expression of a coherent development between material, structure and form, and the realization of a full scale prototype. Theresearch seekstoextend the limits of traditional weaving crafting by pre-computing its configuration and anticipating its morphological and assembly characteristics. In this sense, part of the implicit material computation of 
weaving is designed within the digital environment. A fundamental part of the experiment was the creation of a lightweight self-standing system, capable to partition interior spaces and provides a differential screening experience, to mediate environmental light conditions and user experience. This was instrumental to the development of a low-tech construction system able to enhance antique crafting with digital manufacturing.

\section{Design process}

In the design phase, a methodology for the generation of lightweight weaving structures is developed, allowing the control of the system of holes, precise programming where the wefts are passing, but also generating even stronger friction than traditional weaving, mediating with material constraints such as maximum length and bending resistance.

\section{Programming weaving pattern}

Shifting from baskets to architecture involves a change of scale, and requires the reconsideration of the weaving technique in its dimensional and structural parameters, which cannot be linearly scaled. In two-directional weaving patterns, the overall resistance is achieved by the multiple bending and frictional resistance between the various warp and weft elements. A system of parametrically-controlled holes on the two external edges of each vertical warp element defines a stable and accurate structural system based on frictional resistance. This configuration allows to program precisely the position of the weft elements and generates even stronger friction than traditional weaving. In fact, a script allows to parametrically adjust the dimension and position of the holes on both sides of each rib, mediating between different factors, such as maximum length and bending stiffness of the sticks. By controlling the pattern parameters, different curvatures are imposed to the rattan sticks, and the local stiffness control is enhanced. Consequently, the more the rattan is bent, the higher is the magnitude of force applied on the wooden warps, increasing the friction between the two elements, and in turn the rigidity of the model (Fig.13)

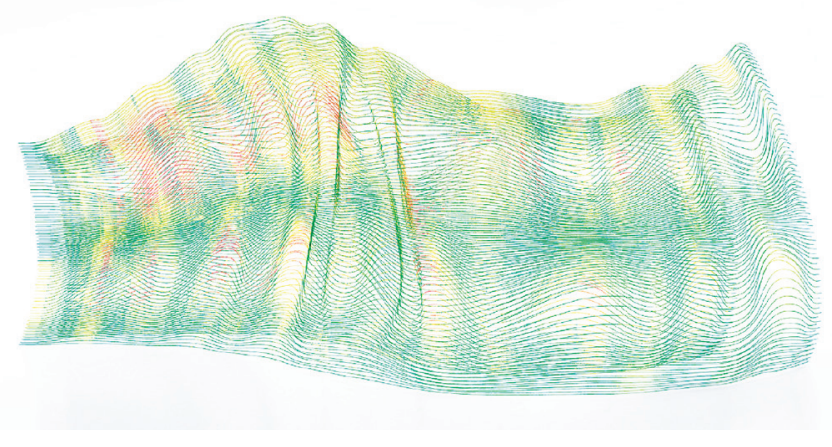

Figure 13: Curvature analysis of wefts in the digital model. The more the rattan is bent the more force is locally applied on the wooden warps, increasing the friction between the warps and wefts.
In traditional weaving systems the plane of action of the warps is mainly horizontal, and consequently the friction generated by the interaction with vertical wefts is constant in position and angle. On the contrary, in the system outlined in this paper, a more complex frictional scheme is generated by the combination of two main aspects: the form of the vertical elements and their disposition, which creates variable curvatures to warp elements in the horizontal direction, and the pattern, which generates variable forces in the vertical direction. Friction forces derive from the contact of the entire section of the strand with the surface of the hole, in contrast to traditional weaving, where only small areas are usually in direct contact (Fig.14). Embedded in the computational model, the control over such a complex system allows the creation and definition of a wide range of different local frictional resistances, both in its amplitude and location. Moreover, the control over the holes disposition is tuned according to extrinsic performative criteria to generate a field with differential screening ratios given by the visual overlap of two external weft sets. By varying the local density of holes along the ribs sides it is possible to vary the density of sticks throughout the whole structure, both controlling local maximum and minimum visual permeability values and generating the overall visual effect. To further increase the possibility of variation in density, a second direction of movement on the plane of the rib has been added perpendicular to the tangent of the rib at each point, which allows a visual superimposition of the strands, amplifying the differential screening field.

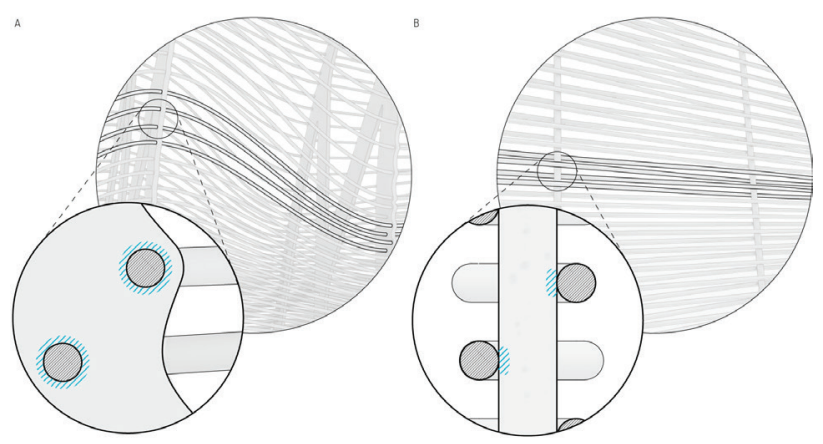

Figure 14: Warp-Weft friction scheme. A. Structural Weaving System allows friction in different areas according to rattan curvature direction; B. Traditional weaving creates a punctual friction between warp and weft elements.

A final prototype, called Weaving Enclosure, has been designed and realized as proof of concept for the design workflow and to test the physical implementation of the construction system (Fig.15). The prototype has been built as the final output of an international research workshop where a joint group of researchers and students have designed, fabricated and built the installation within a building hall that belongs to the Faculty of Architecture and Society. The final design implements the described system with a sinusoidal 
installation of 7.4 by 3.3 meters, with a variable height ranging from 1.8 to 2.9 meters. The lightweight prototype is composed of 79.2 Kilograms of $15 \mathrm{~mm}$ thick poplar plywood warps and 75.7 kilograms of 1.85 kilometers of rattan, divided in 760 different pieces, which range from 1.85 to 3.15 meters in length. The whole structure is assembled in four autonomous modules composed by four vertical ribs each.

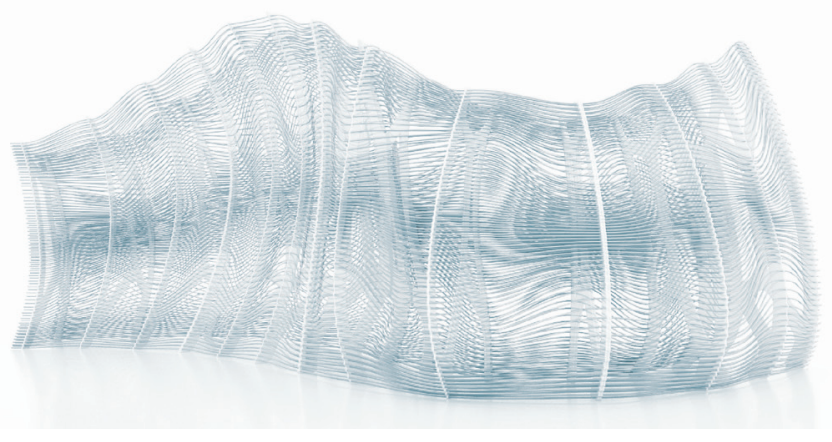

Figure 15: Final design configuration of the Weaving Enclosure. The self-standing structure is 7.4 meters long, 3.3 meters in width and reaches a height of 2.9 meters.

\section{Hybrid fabrication}

A fabrication protocol is developed and processed through an industrial 3-axis CNC milling machine. An optimized .cnc format G-CODE (Fig.16) was generated from Grasshopper to directly provide the machines with pre-processed working trajectories.

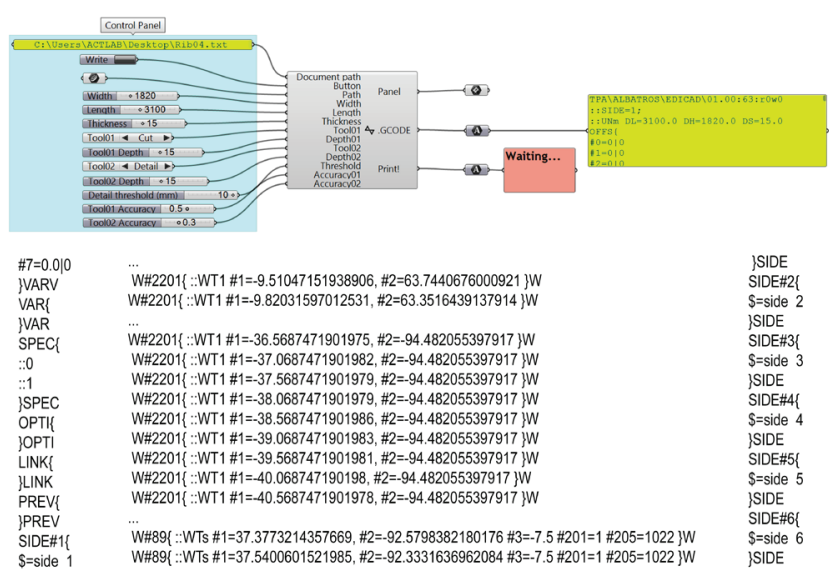

Figure 16: A G-CODE generated from Grasshopper in order to directly provide a CNC machine with pre-processed working trajectories.

\section{Digital crafting}

After the process of fabrication of the warp elements, a manual process of assembly and weaving has been carried out (Fig.17). To fix the programmed shape and keep vertical ribs in position, avoiding movement and deformations, a series of temporary horizontal scaffoldings were designed and $\mathrm{CNC}$ cut to create a statically stable skeleton. After a series of tests, the best technique to weave the 760 rattan sticks proved to be by working on each module separately, weaving the sticks from bottom to top parallel on the two surfaces of each module. Customized holes on the first and on the last rib of each module have been milled to host post-tensioned stick elements fixed by external screws. Hot water has been used to wet the sticks during the weaving process in order to make the fibrous structure of rattan softer, in order to handle the high curvature of sticks needed to obtained and allow it to form smoother curves more easily.

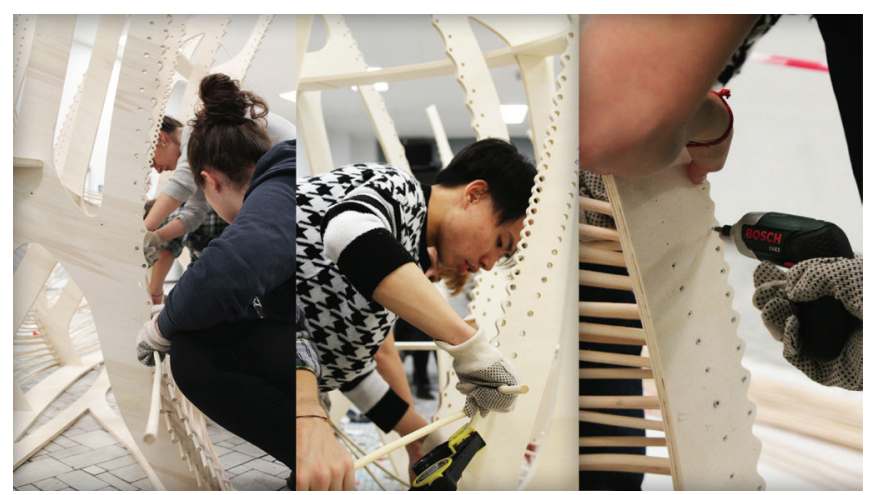

Figure 17: Manual work is still a fundamental step in the weaving process. Right. Screwing process for rattan post-tensioning.

\section{Discussion}

Reiser and Umemoto in Atlas of Novel Tectonics stated that the currently changing notion of architectural design is a consequence of the shift from geometry as an abstract regulator of the materials of construction, to a notion where matter and material behaviours are implicated in geometry itself (Reiser and Umemoto 2006). Following this paradigm, speculative research have promoted a novel material awareness empowered by the advancements in computing and digital manufacturing (Naboni and Paoletti 2015). This paper presents a specific design approach to the material performance and expressivity of weaving, and reinterprets this antique crafting technique as a novel construction system. Integrated computational tools are fundamental to this approach, supporting analysis of non-standard materials and the design of their dynamic behavior. Through this approach, a prototectonic partitioning system has been developed proving the high integration between performance and aesthetics, between the absolute precision of the digital process of design and fabrication, and the implicit imperfection of handcrafts and natural materials. The adopted approach opens interesting perspective for the development of computationally assisted low-tech systems in architecture. 


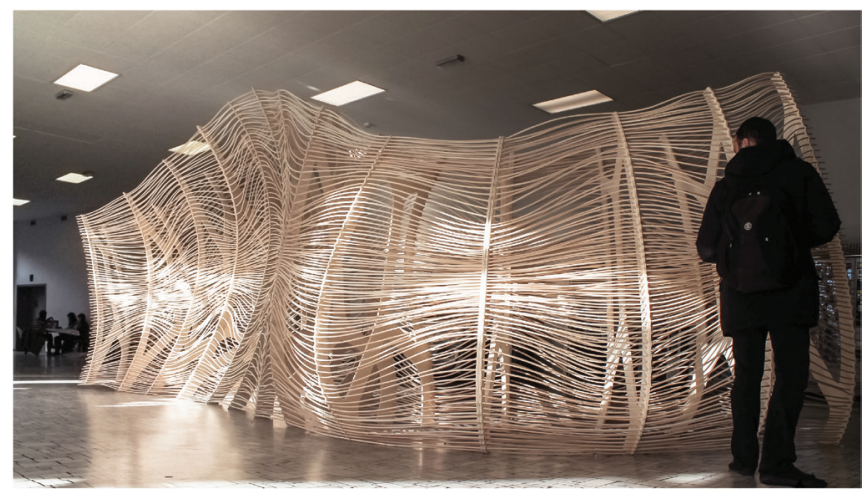

Figure 18: Weaving Enclosure shapes the space and orient the view, creating a variable experience for the user.

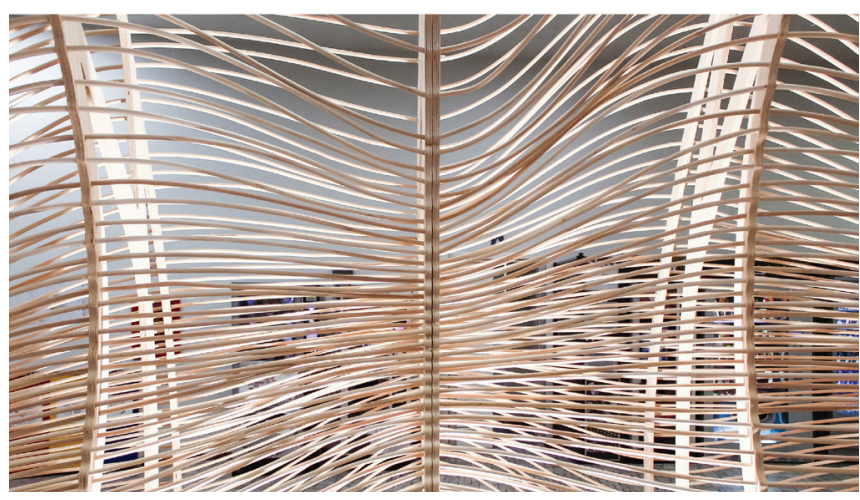

Figure 19: The variable density of rattan wefts generates a differential screening ratio along Weaving Enclosure surfaces.

\section{Acknowledgements}

We wish to thank Prof. Ingrid Paoletti and our colleagues from ACTLAB for their support of this project, in particular Bernardo de Cossio who conducted initial tests and research on materials; Paolo Alborghetti and the students participating in UnExpected MateReality Workshop 2014. We express our gratitude to GD Guidetti e Dassi for supporting the $\mathrm{CNC}$ fabrication and Laboratorio Prove Materiali and Laboratorio Modelli e Prototipi of Politecnico di Milano.

\section{References}

Dransfield, J., Tesoro, F. and Manokaran, N. (2000). Rattan: current research issues and prospects for conservation and sustainable development. Food and Agriculture Organization of the United States.

Eichenseer, C., Will, C., Rampf, M., Wend, S. and Greil P. (2009). Biomorphous porous hydroxyapatite-ceramics from rattan (Calamus Rotang). Journal of Materials Science: Materials in Medicine. New York: Springer US, 21(1), 131137.

Griffen, C. S. (2001). Weaving as an Analogy for Architectural Design. In Paradoxes of Progress: Architecture and
Education in a Post-utopian Era. Proceedings, 89th ACSA Annual Meeting. 478-483.

Hensel, M., Menges, A. and Weinstock, W. (2010). Emergent Technologies and Design: Towards a Biological Paradigm for Architecture. New York: Routledge.

Hensel, M (2013). Performance-Oriented Architecture: Rethinking Architectural Design and the Built Environment. London: John Wiley \& Sons

Menges, A. (2012). Material Resourcefulness. Activating Material Information in Computational Design. In Material computation. Higher integration in morphogenetic design, ed. Menges, A., AD, Architectural Design, London: John Wiley \& Sons, 216, 36.

Muslimin, R. (2010). Learning from Weaving for Digital Fabrication in Architecture. Leonardo, 43 (4), 340-349.

Naboni, R. and Paoletti, I. (2015). Advanced Customization in Architectural Design and Construction. New York: Springer US

Obilade, I.O., Olutoge, F.A. (2014). Flexural Characteristics of Rattan Cane Reinforced Concrete Beams. The International Journal Of Engineering And Science (IJES), 3 (6), 38-42. Otto, F. (1969). Minimal Nets. Institute for Lightweight Structures, University of Stuttgart, Stuttgart : K. Krämer. Oxman, N. (2007). Digital Craft: Fabrication Based Design in the Age of Digital Production. Workshop Proceedings for Ubicomp 2007: International Conference on Ubiquitous Computing. September; Innsbruck, Austria, 534-538.

Oxman, N. (2010). Material-based design computation. Thesis (Ph. D.). Cambridge: Massachusetts Institute of Technology, Dept. of Architecture.

Ranjan, M. P. (1985). Structure of Bamboo Baskets. A classification of forming processes, weave structures and significant details. In IL-35 Bambus - Bamboo, ed. by Gab, S., Drusedau, H. \& Hennike, J. Stuttgart: Institute fur Leichte Fachentragwerke, 356 - 367.

Reiser J, Umemoto N (2006). Atlas of novel tectonics. New York: Princeton Architectural Press.

Saunders, R. (1990). Wicker Furniture: A Guide to Restoring and Collecting. Three Rivers Press.

Semper, G. and Mallgrave, F.H. (1989). The Four Elements of Architecture: and Other Writings. Cambridge: Cambridge University Press.

Thomsen, M. R. and Tamke, M. (2014). Digital Crafting: Performative Thinking for Material Design. In Inside Smartgeometry: Expanding the Architectural Possibilities of Computational Design. eds. Peters, B. and Peters, T, London: John Wiley \& Sons. 243-253.

Tomlinson, P.B., Fisher, J.B., Spangler R.E. and Richer R.A. (2001). Stem Vascular Architecture in the Rattan Palm Calamus (Arecaceae-Calamoideae-Calaminae). American Journal of Botany 88(5): 797-809.

Wikipedia. Basket weaving.

http://en.wikipedia.org/wiki/Basket_weaving 